Reviving the Method of Particular Solutions

Betcke, Timo and Trefethen, Lloyd N.

2005

MIMS EPrint: 2006.365

Manchester Institute for Mathematical Sciences

School of Mathematics

The University of Manchester

\footnotetext{
Reports available from: http://eprints.maths.manchester.ac.uk/

And by contacting: The MIMS Secretary

School of Mathematics

The University of Manchester

Manchester, M13 9PL, UK
} 


\title{
Reviving the Method of Particular Solutions*
}

Timo Betcke ${ }^{\dagger}$

Lloyd N. Trefethen ${ }^{\dagger}$

\begin{abstract}
Fox, Henrici, and Moler made famous a "method of particular solutions" for computing eigenvalues and eigenmodes of the Laplacian in planar regions such as polygons. We explain why their formulation of this method breaks down when applied to regions that are insufficiently simple and propose a modification that avoids these difficulties. The crucial changes are to introduce points in the interior of the region as well as on the boundary and to minimize a subspace angle rather than just a singular value or a determinant. Similar methods may be used to improve other "mesh-free" algorithms for a variety of computational problems.
\end{abstract}

Key words. eigenvalues, method of particular solutions, subspace angles

AMS subject classifications. $65 \mathrm{~N} 35,65 \mathrm{~F} 22,65 \mathrm{~N} 25$

DOI. $10.1137 / \mathrm{S} 0036144503437336$

I. Introduction. Every reader of SIAM Review is probably familiar with the MATLAB logo, which depicts the first eigenmode of the Laplace operator on an Lshaped region (Figure 1.1). The historical roots of this image are easy to identify. In 1967 Fox, Henrici, and Moler (FHM) published a beautiful article, "Approximations and Bounds for Eigenvalues of Elliptic Operators," that described the method of particular solutions (MPS) and presented numerical examples [11]. The algorithm, built on earlier work by Bergman [2] and Vekua [28], makes use of global expansions and collocation on the boundary. The central example in the FHM paper was the Lshaped region, and the authors' enthusiasm for this subject can be seen in the related papers and dissertations published around this time by Fox's and Mayers' students Donnelly, Mason, Reid, and Walsh at Oxford [8, 19, 25], and Moler's students Schryer and Eisenstat at Michigan and Stanford [10,26]. When Moler developed the first version of MATLAB a decade later, one of the first applications he tried was the $\mathrm{L}$ shape, which lives on in the logo and membrane commands in MATLAB today ${ }^{1}$ and as an example in Moler's new textbook [22].

* Received by the editors November 5, 2003; accepted for publication (in revised form) August 10, 2004; published electronically July 29, 2005.

http://www.siam.org/journals/sirev/47-3/43733.html

${ }^{\dagger}$ Computing Laboratory, Oxford University, Wolfson Building, Parks Road, Oxford OX1 3QD, UK (timob@comlab.ox.ac.uk, LNT@comlab.ox.ac.uk).

${ }^{1}$ Most MATLAB users probably don't know that the famous logo image is an eigenfunction. Among those who do, probably most have not noticed that it fails to satisfy the intended zero boundary conditions! For aesthetic reasons, Moler chose this incorrect image instead of a correct one, which can be obtained by replacing the default membrane $(1,15,9,2)$ by membrane $(1,15,9,4)$. Neither membrane nor logo computes eigenvalues; they work with values previously computed and stored as constants. 


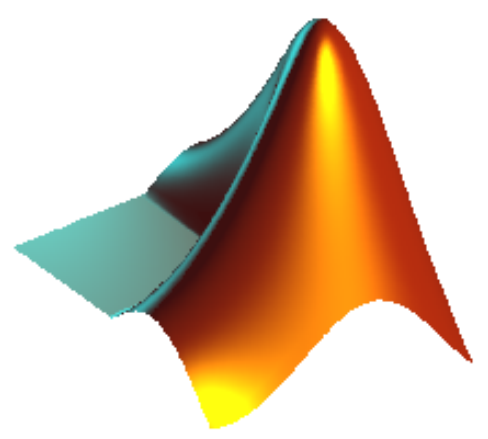

Fig. I.I The MATLAB logo: first eigenmode of an L-shaped region (see footnote 1). In section 5 we calculate the corresponding eigenvalue to 14 digits of accuracy as 9.6397238440219. (The L-shaped membrane logo is a trademark and MATLAB ${ }^{\circledR}$ is a registered trademark of The MathWorks, Inc., used with permission.)

After the early 1970s, the MPS got less attention. It would seem that the main reason for this may be that the method runs into difficulties when dealing with all but the simplest regions. Indeed, the MPS has trouble even with the L shape itself, unless one reduces the problem to a square of one-third the size by the use of certain symmetries, as FHM did. ${ }^{2}$ Instead, other methods for these problems have come to the fore. The state of the art at present is a method developed by Descloux and Tolley [7] and improved by Driscoll [9] based on local expansions near each vertex rather than a single global expansion.

It puzzled us that such a simple and elegant method should have run into difficulties that were not well understood. We have examined the behavior of the classic MPS and have found that the root of the problem is that, working as it does only with points along the boundary of the domain, it fails to impose effectively the condition that the eigenfunction should be nonzero in the interior. As a result it is undone by the existence of functions in the search space that are close to zero everywhere, in the interior as well as on the boundary. We have devised a modified MPS method to get around this problem, and the aim of this paper is to present this method. We believe that the MPS is now robust enough to deal with quite complicated regions, and indeed, for computing eigenvalues on polygons, it may be competitive with any other method. It also appears that the ideas we propose here, with suitable modifications, may be applicable to other numerical algorithms involving expansions in ill-conditioned bases. We hope to report on such generalizations in the future.

The focus of this paper is on robust extraction of eigenvalues and eigenvectors from subspaces. In addition, the effectiveness of the MPS depends on the quality of those subspaces - a matter of approximation theory that is nontrivial when there is more then one singular corner. These issues of approximation will be considered in a separate article.

\footnotetext{
${ }^{2} \mathrm{~A}$ curious piece of history arises here. A year before the appearance of [11], Kac had published his celebrated paper, "Can One Hear the Shape of a Drum?" [15], which stimulated great interest in planar Laplace eigenvalue problems and a great deal of research activity among pure mathematicians. Twenty-six years later Gordon, Webb, and Wolpert showed that the answer is no, one cannot hear the shape of a drum [12]. To widespread surprise, their proof was elementary; it used the same kinds of symmetries known for many years and employed by FHM. An eloquent proponent of the use of such symmetries, who certainly influenced Fox et al., was Joseph Hersch of the ETH in Zurich [14].
} 
2. The Laplace Eigenvalue Problem in the Plane. In this section we review some properties of the Laplace eigenvalue problem with Dirichlet boundary conditions,

$$
\begin{aligned}
-\Delta u & =\lambda u \quad \text { in } P, \\
u=0 & \text { on } \partial P,
\end{aligned}
$$

where $P$ is a bounded domain in the plane. These types of problems arise in the treatment of vibrations of a membrane, where the eigenvalue $\lambda$ is the square of the frequency of vibration $\omega$. An excellent survey of (2.1) has been published by Kuttler and Sigillito [17]. Here we restrict ourselves to some fundamental results.

All eigenvalues of (2.1) are positive. We can order them with multiplicity according to

$$
0<\lambda_{1} \leq \lambda_{2} \leq \cdots \leq \lambda_{k} \leq \cdots,
$$

with a limit point at infinity, and the corresponding eigenfunctions can be chosen to form an orthonormal complete set in $L_{2}(P)$. That is,

$$
\int_{P} u_{i} u_{j} \mathrm{dx}=\delta_{i j}
$$

where $\delta_{i j}$ is the Kronecker delta.

Elementary solutions can be obtained for some simple domains. For a rectangle with $0 \leq x \leq a, 0 \leq y \leq b$ the eigenfunctions are

$$
u_{m, n}(x, y)=\sin \left(\frac{m \pi x}{a}\right) \sin \left(\frac{n \pi y}{b}\right), \quad m, n=1,2, \ldots,
$$

with corresponding eigenvalues

$$
\lambda_{m, n}=\pi^{2}\left[\left(\frac{m}{a}\right)^{2}+\left(\frac{n}{b}\right)^{2}\right] .
$$

In the case of an unbounded wedge with interior angle $\pi / \alpha$, separation of variables leads to the solution

$$
u(r, \theta)=J_{\alpha k}(\sqrt{\lambda} r) \sin \alpha k \theta, \quad k \in \mathbb{N} \backslash\{0\},
$$

for any $\lambda>0$, where $J_{\alpha k}$ is a Bessel function (Figure 2.1). The spectrum is continuous; it is the restriction to a bounded domain that makes it discrete. For example, in the case of a disk of radius $a$ the eigenfunctions are given by

$$
u_{m, n}(r, \theta)=J_{m}\left(\frac{j_{m n} r}{a}\right)[A \cos m \theta+B \sin m \theta], \quad m=0,1, \ldots, n=1,2, \ldots,
$$

where $j_{m n}$ is the $n$th zero of $J_{m}$. The eigenvalues are

$$
\lambda_{m, n}=\left(\frac{j_{m n}}{a}\right)^{2} .
$$

The eigenvalues of (2.1) cannot be arbitrarily distributed. An important result to this effect is Weyl's law,

$$
\lambda_{n} \sim \frac{4 \pi n}{A} \text { as } n \rightarrow \infty
$$




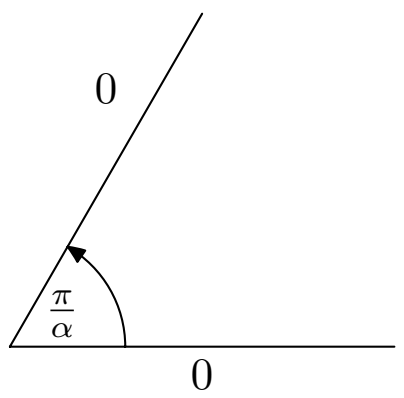

Fig. 2.I A wedge with interior angle $\pi / \alpha$, on which the eigenfunctions are explicitly known as Fourier-Bessel functions (2.2).

where $A$ is the area of $P$. A proof can be found, for example, in [5, pp. 429-442]. The nodal lines of $u_{n}$ are the set of points in $P$ where $u_{n}=0$. Courant's nodal line theorem states that the nodal lines of the $n$th eigenfunction $u_{n}$ divide $P$ into not more than $n$ subdomains [5, p. 452]. The eigenfunction of the first eigenvalue $\lambda_{1}$ has no nodal lines, and by orthogonality it follows that $\lambda_{1}$ is always simple.

The eigenfunctions of (2.1) are infinitely differentiable in $P$ and continuous on $P \cup \partial P$. An eigenfunction $u_{n}$ can be reflected as a $\mathcal{C}^{\infty}$ function across any part of the boundary that is analytic. At a corner where $\partial P$ consists locally of two line segments meeting at the angle $\pi / k$ for an integer $k, u_{n}$ can be extended to a $\mathcal{C}^{\infty}$ function in a whole neighborhood of the corner by reflecting $k-1$ times across the boundary to obtain a function that is $\mathcal{C}^{\infty}$ in a region whose boundary contains a line segment with no corner, followed by one final reflection across this line segment [5, p. 395]. Throughout this paper we will call corners of a polygon regular if their angles are integer fractions of $\pi$. All other corners will be called singular. At singular corners it is not possible to continue the eigenfunction analytically to a whole neighborhood of the corner. This can be seen from the argument of analytic continuation or by looking at the asymptotic behavior of the eigenfunction near the corner. In [18] it is shown that if $u_{n}$ is nonzero, there is a nonzero constant $\gamma_{n}$ such that

$$
u_{n}=\gamma_{n} r^{\alpha} \sin \alpha \theta+o\left(r^{\alpha}\right) .
$$

Clearly if $\alpha \notin \mathbb{N}$, the eigenfunction has a branch point at the corner. To obtain rapidly converging methods, these singularities have to be dealt with.

3. The Method of Particular Solutions. The idea of the method of particular solutions (MPS) starts by considering various solutions of the eigenvalue equation (2.1a) for a given value of $\lambda$. One then tries to vary $\lambda$ until one can find a linear combination of such solutions that satisfies the boundary condition $(2.1 \mathrm{~b})$ at a number of sample points along the boundary.

From (2.2) we see that a convenient set of particular solutions near a corner of angle $\pi / \alpha$ are the functions

$$
u^{(k)}(r, \theta)=J_{\alpha k}(\sqrt{\lambda} r) \sin \alpha k \theta ;
$$

we call these Fourier-Bessel functions. The advantage of these functions is that not only do they satisfy (2.1a), they also satisfy (2.1b) along the adjacent line segments. 


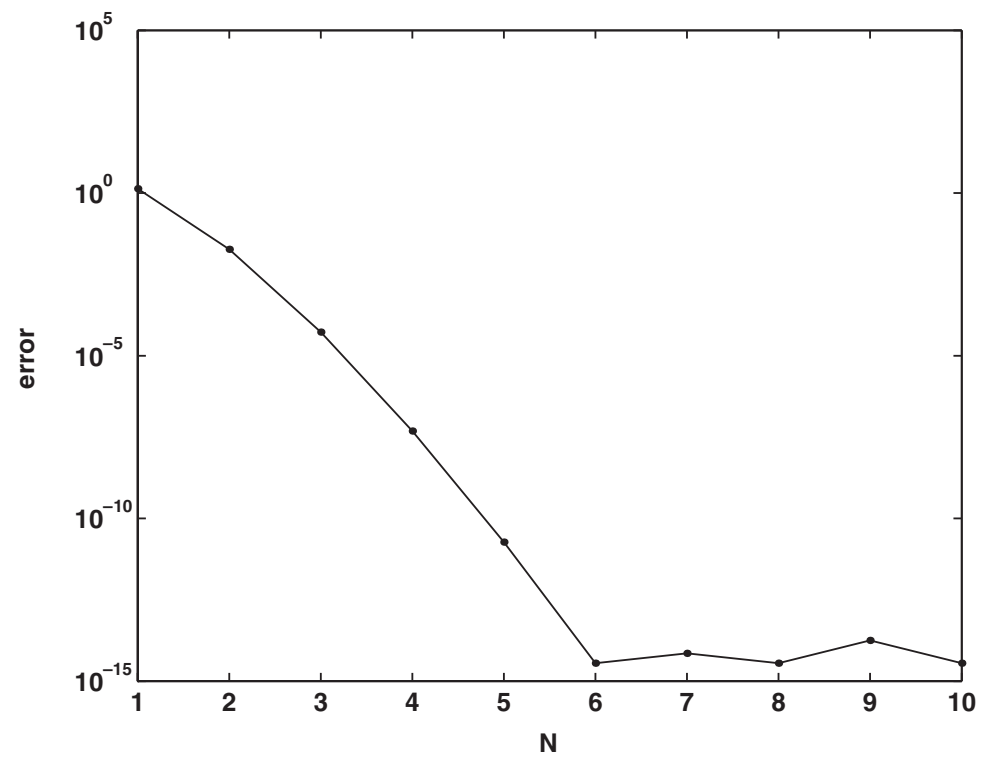

Fig. 3.I Convergence to the first eigenvalue of the unit square with the FHM method. The parameter $N$ denotes the number of collocation points on each of the two sides not adjacent to the corner at which the expansion is based.

We consider approximate eigenfunctions

$$
u^{*}(r, \theta)=\sum_{k=1}^{N} c_{k}^{(N)} u^{(k)}(r, \theta)
$$

with parameters $c_{j}^{(N)}$ to be determined so as to attempt to satisfy $(2.1 \mathrm{~b})$ also on the remainder of the boundary. One approach is to let $\left(r_{i}, \theta_{i}\right)$ be $N$ collocation points on the boundary and require $u^{*}\left(r_{i}, \theta_{i}\right)=0$ for $i=1, \ldots, N$. This amounts to considering the square system of nonlinear equations

$$
A(\lambda) c=0
$$

with

$$
a_{i k}(\lambda)=J_{\alpha k}\left(\sqrt{\lambda} r_{i}\right) \sin \alpha k \theta_{i}, \quad i, k=1, \ldots, N .
$$

We can attempt to solve these equations by varying $\lambda$ and looking for a zero of $\operatorname{det} A(\lambda)$. This is the approach proposed in the FHM paper. Alternatively, we can take more sample points than expansion terms. Then (3.3) becomes a rectangular system, and we could attempt to solve it by varying $\lambda$ and looking for a zero or a near-zero of the smallest singular value of $A(\lambda)$. This generally superior approach was investigated by Moler in a technical report in 1969 [21].

Figure 3.1 shows the convergence history for the FHM method for computing the first eigenvalue $2 \pi^{2}$ of the unit square with an expansion around one corner and collocation points equally spaced along the opposite two sides. The results obtained by this method were taken to be the zeros of $\operatorname{det} A(\lambda)$ closest to $2 \pi^{2}$. The figure reveals 


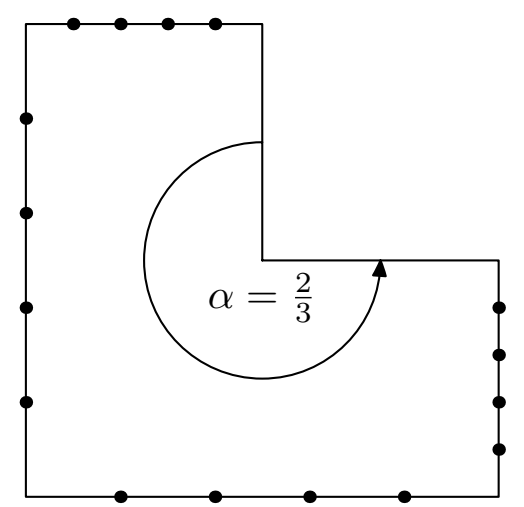

Fig. 3.2 The L-shaped membrane, with $N$ collocation points equally spaced along each side not adjacent to the reentrant corner (here $N=4$ ).

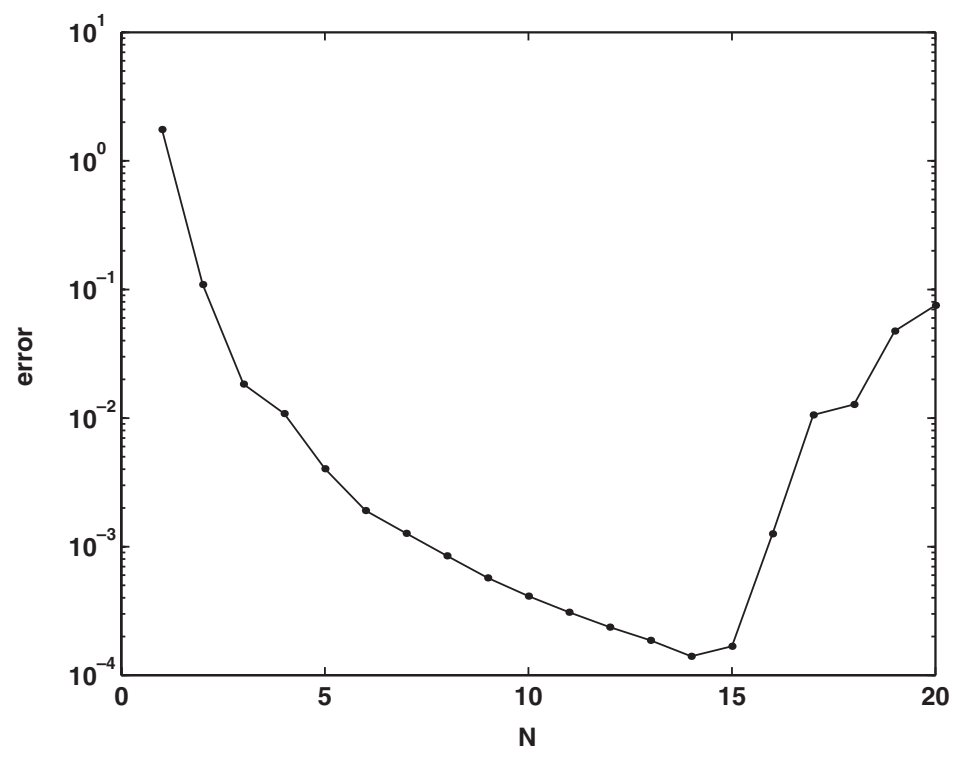

Fig. 3.3 The error of FHM for the first eigenvalue of the L-shaped domain. Convergence breaks down after $N=14$, and one never gets more than four digits of accuracy.

"spectral" convergence, that is, convergence at the rate $O\left(N^{-s}\right)$ for every $s$, and the method seems to be working quite well. However, this example is too simple to reveal much about the FHM method. So let us look at the famous L shape. This domain has one singular corner, the reentrant corner. To catch this singularity, expansions around the reentrant corner are used with collocation again in $N$ equally spaced points along each of the nonadjacent sides (Figure 3.2). Figure 3.3 shows the convergence behavior for the first eigenvalue $\lambda_{1} \approx 9.6397238440219$. The FHM method fails to obtain more than four digits of accuracy, breaking down after $N=14$. Fox, Henrici, and Moler got eight digits, but this success depended on their use of symmetries to reduce the domain to a square. 


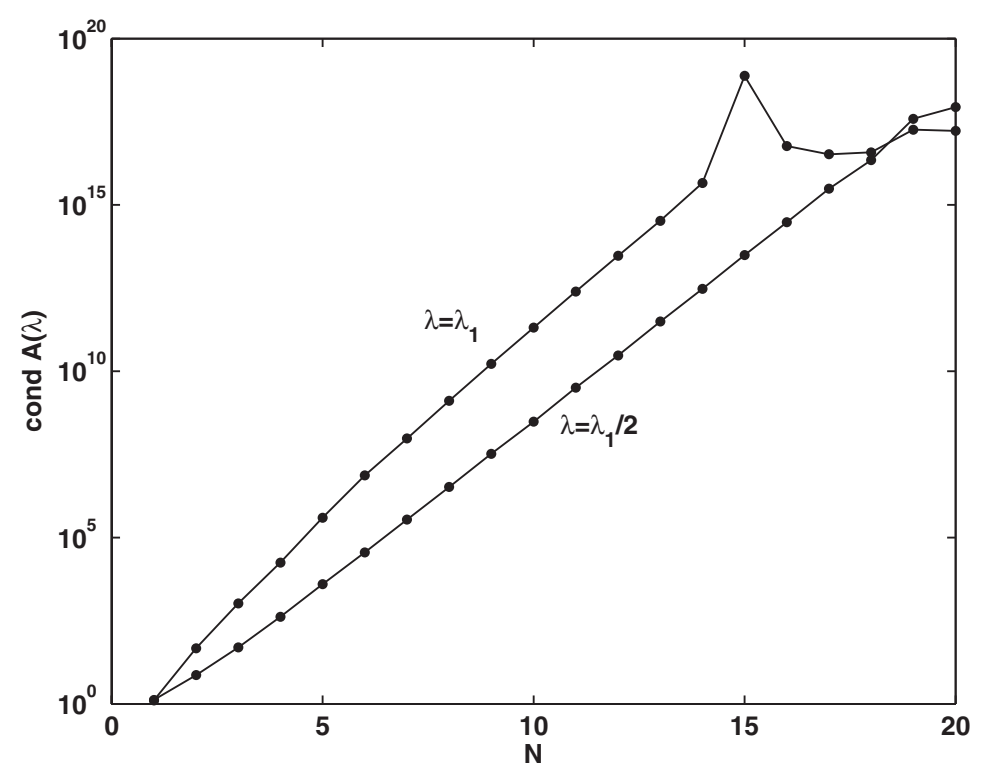

Fig. 4.I The condition number of $A(\lambda)$ for different parameters $N$ determining the number of boundary collocation points (4N). The classic MPS fails because $A(\lambda)$ approaches singularity nearly as fast when $\lambda$ is not an eigenvalue $\left(\lambda=\lambda_{1} / 2\right)$ as when it is an eigenvalue $\left(\lambda=\lambda_{1}\right)$.

4. Failure of the MPS. What has gone wrong? We can explain the results of Figure 3.3 as follows. The aim of the FHM method is to find a value $\lambda$ for which there exists a nontrivial linear combination of Fourier-Bessel functions (3.1) that is zero at the boundary collocation points. If $\lambda$ is not close to an eigenvalue of (2.1), we expect the collocation equation (3.3) to have no nontrivial solution. However, in general the Fourier-Bessel basis behaves similarly to a power basis of monomials $z^{k}$, and the condition number of $A(\lambda)$ grows exponentially as more basis terms are added. This happens for any value $\lambda$, whether or not it is an eigenvalue. Consequently, when $N$ is large, it is always possible to find linear combinations of the columns of $A(\lambda)$ that are close to zero, regardless of the value of $\lambda$. If $\lambda$ is not close to an eigenvalue, this results in approximating the zero function on the domain $P$. But since the FHM method examines only boundary points, it does not distinguish between a true eigenfunction and the zero function.

To demonstrate this effect, Figure 4.1 plots cond $A(\lambda)$ for the L-shaped domain as a function of $N$ (the columns of $A(\lambda)$ were scaled to unit length). The curve for $\lambda=\lambda_{1}$ uses the approximation $\lambda_{1} \approx 9.6397238440219$ for the true first eigenvalue on the L-shaped domain (section 5). The second curve uses the arbitrary value $\lambda_{1} / 2$, not close to an eigenvalue. Both curves grow exponentially as $N$ increases, and the gap between them, on which the FHM method depends for locating eigenvalues, does not widen much. After $N=14$ the results become erroneous due to rounding errors; $A(\lambda)$ is numerically singular for both the true eigenvalue and the spurious one.

Various changes can be made to the FHM method to get a few more digits for the L-shaped region. If the points on the boundary are distributed in a Chebyshev rather than equispaced manner, then with sufficient care one can get up to eight digits. If one 
moves to a rectangular matrix one gets a very fragile optimization problem, which becomes somewhat better behaved if each column of $A(\lambda)$ is scaled to unit norm before one minimizes the singular value. With sufficiently careful optimization one can get many digits with this formulation, but the objective function to be minimized is troublesome, consisting of a uniformly low value with tiny spikes down to nearly zero that have to be located; as $N \rightarrow \infty$ these spikes get narrower and the problem gets rapidly more difficult.

None of these ideas seems capable of making the FHM approach effective for more complicated regions, especially those with more than one singular corner, as we shall treat in later sections. For such regions, the phenomenon illustrated in Figure 4.1 is typical: the matrix $A(\lambda)$, whether square or rectangular, becomes exponentially more ill-conditioned for all values of $\lambda$ as $N$ increases. In practice it quickly becomes impossible to locate eigenvalues.

We are not the first to note that there are difficulties with the classical form of the MPS. Fox, Henrici, and Moler wrote:

In all fairness, it should be reported that results are not always as satisfactory as these examples indicate.... Other methods... are currently being investigated.

And Driscoll [9], attempting to apply the method to more challenging regions, encountered just the problem we have described:

As the number of terms in the truncated expansion is increased, the matrix becomes very nearly singular for all values of $\lambda$, and detecting the true singularity numerically becomes impossible. In fact, we have been unable to produce more than two or three accurate digits for a few of the smallest eigenvalues [of the GWW isospectral drums considered below in section 7] with this method.

5. A Modified Method. In this section we introduce a modified approach that overcomes this problem. The basic idea of the improved method is to restrict the set of admissible functions to functions that are bounded away from zero in the interior. In the next section we will show that this idea is equivalent to the minimization of the angle between the space of functions that satisfy the eigenvalue equation and the space of functions that are zero on the boundary.

We have seen that the boundary points alone do not deliver enough information to decide if an approximate eigenfunction is spurious. To get around this problem we add additional interior points. Let $m_{B}$ be the number of boundary points and $m_{I}$ the number of interior points. Our discrete sample of an approximate eigenfunction is the vector $u \in \mathbb{R}^{m}, m=m_{B}+m_{I}$, of the function evaluated at those points. For convenience, we order the elements of $u$ so that the first $m_{B}$ components correspond to the boundary and the remaining $m_{I}$ to the interior. The matrix $A(\lambda)$ of the last section now has more rows,

$$
A(\lambda)=\left[\begin{array}{c}
A_{B}(\lambda) \\
A_{I}(\lambda)
\end{array}\right]
$$

with $B$ and $I$ corresponding to boundary and interior (see Figure 5.1).

By $\mathcal{A}(\lambda) \subset \mathbb{R}^{m}$ we denote the range of $A(\lambda)$, that is, the space of trial functions sampled at boundary and interior points. An orthonormal basis of $\mathcal{A}(\lambda)$ can be 


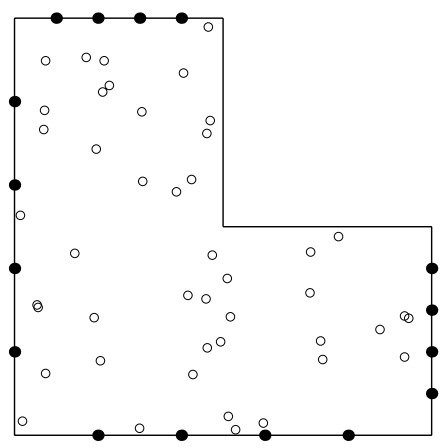

Fig. 5.I In the new method the Fourier-Bessel functions are sampled at interior as well as boundary points. The interior points are chosen randomly.

constructed by a QR factorization of $A(\lambda)$,

$$
Q(\lambda)=\left[\begin{array}{l}
Q_{B}(\lambda) \\
Q_{I}(\lambda)
\end{array}\right]
$$

Each unit vector $u \in \mathcal{A}(\lambda)$ has the form

$$
u=Q(\lambda) v=\left[\begin{array}{l}
Q_{B}(\lambda) \\
Q_{I}(\lambda)
\end{array}\right] v, \quad\|v\|=1, \quad v \in \mathbb{R}^{N} .
$$

Since we are interested in vectors that are small at the boundary points, it is natural to consider the constrained minimization problem of finding the vector $v$ that minimizes the part of $u$ belonging to the boundary:

$$
\min _{v \in \mathbb{R}^{N},\|v\|=1}\left\|Q_{B}(\lambda) v\right\| .
$$

The vector $\tilde{v}$ which solves (5.2) is the right singular vector corresponding to the smallest singular value of $Q_{B}(\lambda)$, which we denote by $\sigma(\lambda)$. Hence,

$$
\sigma(\lambda)=\min _{v \in \mathbb{R}^{N},\|v\|=1}\left\|Q_{B}(\lambda) v\right\|=\left\|Q_{B}(\lambda) \tilde{v}\right\| .
$$

For the norm of the corresponding vector $\tilde{u}=Q \tilde{v}$ we have

$$
1=\|\tilde{u}\|^{2}=\left\|\left[\begin{array}{l}
Q_{B}(\lambda) \\
Q_{I}(\lambda)
\end{array}\right] \tilde{v}\right\|^{2}=\sigma(\lambda)^{2}+\left\|Q_{I}(\lambda) \tilde{v}\right\|^{2} .
$$

Therefore, an approximate eigenfunction that is small on the boundary points is automatically close to unit norm on the interior points, and spurious solutions are excluded.

Figure 5.2 shows $\sigma(\lambda)$ for varying values of $\lambda$ in the case of the L-shaped domain. On each side nonadjacent to the reentrant corner 50 equally spaced boundary points were used. In the interior we take 50 randomly distributed points (the same for all values of $\lambda$ ). The columns of $A(\lambda)$ contain samples of the first 15 Fourier-Bessel functions. The graph shows minima of $\sigma(\lambda)$ close to the first three eigenvalues of (2.1). 


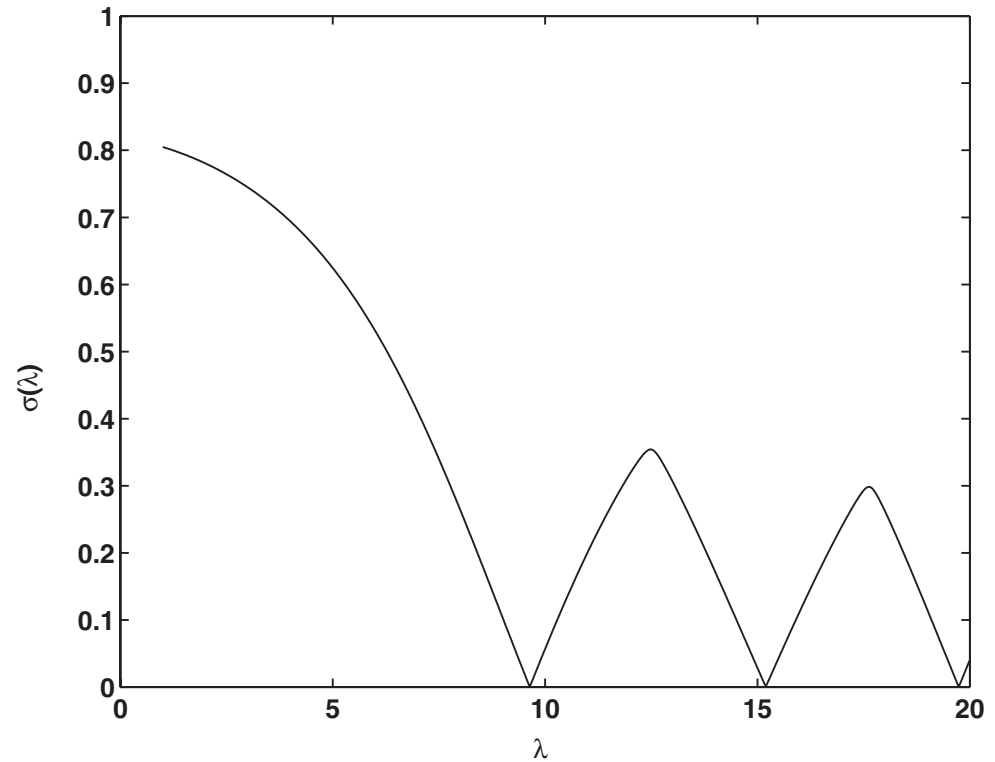

Fig. 5.2 The function $\sigma(\lambda)$ in the case of the L-shaped domain $(N=15)$.

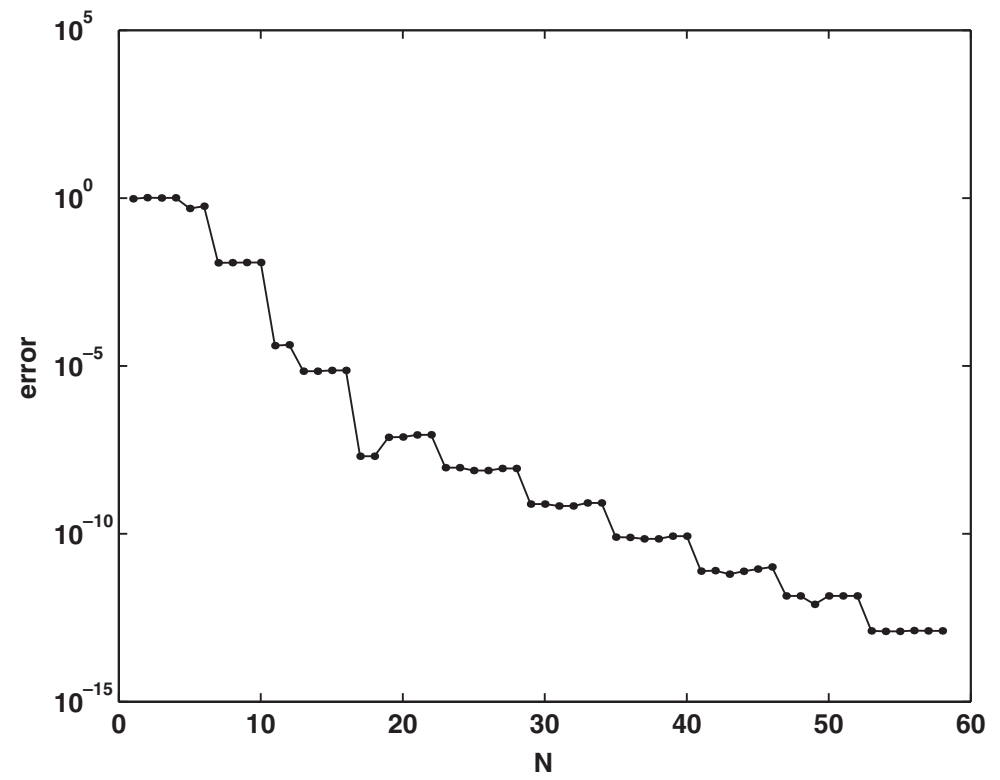

Fig. 5.3 The new method achieves 14 digits of accuracy for the first eigenvalue of the L-shaped domain. Compare Figure 3.3.

The convergence curve for the first eigenvalue is shown in Figure 5.3; as the "exact" solution we took the solution obtained with $N=60$. The jumps in the error curve are caused by the fact that for reasons of symmetry, not all Fourier-Bessel 


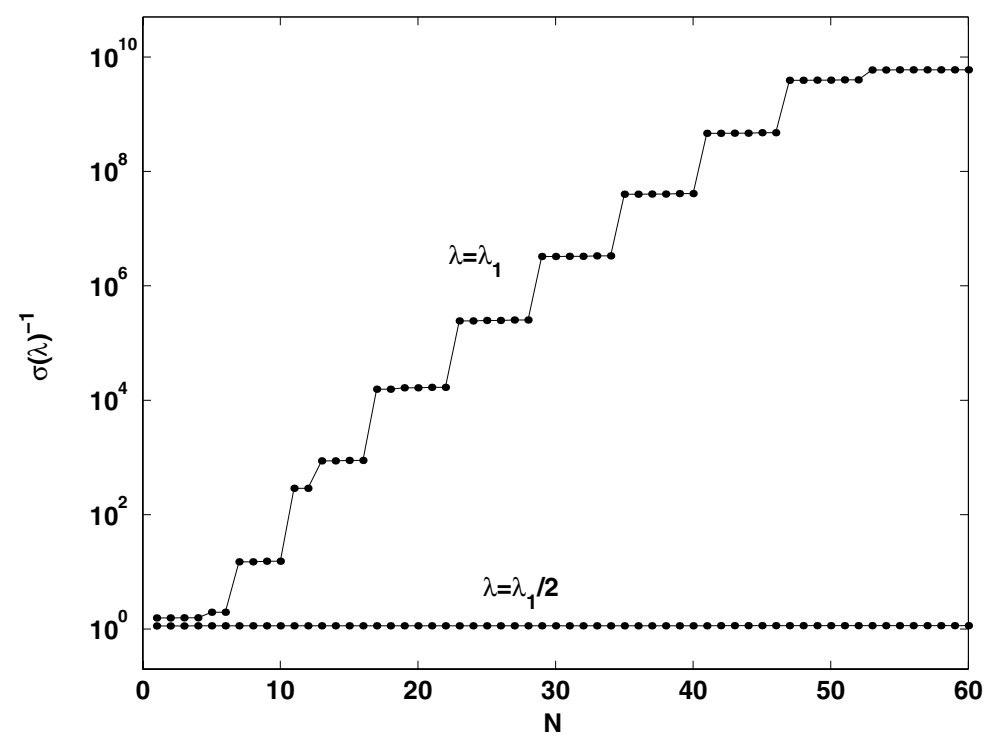

Fig. 5.4 Repetition of Figure 4.1 but for $Q_{B}(\lambda)$ instead of $A(\lambda)$. Now there is a clear separation, the basis of our modified algorithm, between $\lambda=\lambda_{1}$ and $\lambda=\lambda_{1} / 2$.

terms contribute to the series approximation of the eigenfunction [11]; the reason the plateaus are not perfectly flat is that the randomly located interior sample points perturb this symmetry. The minima of $\sigma(\lambda)$ are computed with the MATLAB function fminsearch. We obtain 10 digits of accuracy at $N=41$ and 14 digits of accuracy with a value of 9.6397238440219 at $N=60$. This is a decisive improvement over the 4 digits of accuracy in Figure 3.3. In section 7 we will present several examples of the new method involving more complicated domains with several singularities.

The new method finds eigenvalues of (2.1) by looking for values of $\lambda$ for which $Q_{B}(\lambda)$ becomes nearly singular. Figure 5.4, following Figure 4.1, plots the condition number of this matrix as $N$ increases for $\lambda=\lambda_{1}$ and $\lambda=\lambda_{1} / 2$. The curve for $\lambda_{1}$ grows exponentially as $N$ increases, but the curve for $\lambda_{1} / 2$ stays close to 1 .

6. The Relationship to Subspace Angles. In this section we give a geometric interpretation of the new method, a connection between the constrained minimization problem (5.2) and angles between certain subspaces.

The angle between two subspaces $\mathcal{F}$ and $\mathcal{G}$ is defined by

$$
\cos \measuredangle(\mathcal{F}, \mathcal{G})=\sup _{\substack{u \in \mathcal{F},\|u\|=1 \\ v \in \mathcal{G},\|v\|=1}}\langle u, v\rangle .
$$

In [4] Björck and Golub give a detailed discussion of angles between subspaces and how to compute them numerically.

The space $\mathcal{A}(\lambda)$ consists of samples of functions that satisfy the eigenvalue equation (2.1a) but not necessarily the boundary condition (2.1b). Now let $\mathcal{D}_{0} \subset \mathcal{R}^{m}$ be the space of vectors that are zero at the boundary points, i.e., samples of functions that satisfy (2.1b) but not necessarily (2.1a). The discretely sampled eigenvalue problem then has a nontrivial solution if and only if $\mathcal{A}(\lambda)$ and $\mathcal{D}_{0}$ have a nontrivial 


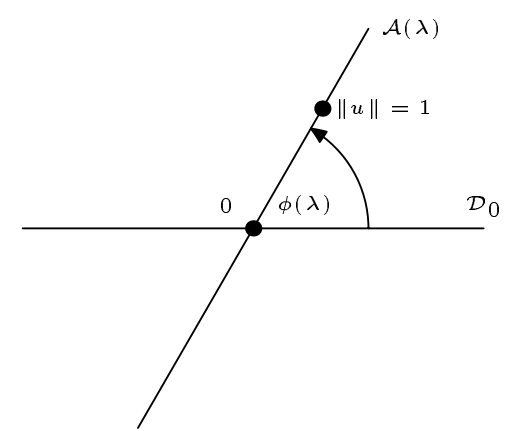

Fig. 6.I Geometric interpretation of the new algorithm. We consider the angle between the spaces of functions that satisfy the eigenvalue equation $(\mathcal{A}(\lambda))$ and the boundary conditions $\left(\mathcal{D}_{0}\right)$. $\lambda$ is an eigenvalue if and only if this angle is zero.

intersection, i.e.,

$$
\phi(\lambda):=\measuredangle\left(\mathcal{A}(\lambda), \mathcal{D}_{0}\right)=0 .
$$

This is easily seen to be equivalent to the condition $\sigma(\lambda)=0$, where $\sigma(\lambda)$ is the singular value defined in (5.3). More generally, we have an equivalence between singular values and subspace angles even when they are nonzero.

THEOREM 6.1. For all $\lambda>0$, the singular value $\sigma(\lambda)$ of (5.3) satisfies

$$
\sigma(\lambda)=\sin \phi(\lambda)=\sin \measuredangle\left(\mathcal{A}(\lambda), \mathcal{D}_{0}\right) .
$$

Proof. Let $P_{D}$ be the orthogonal projector onto the space $\mathcal{D}_{0}$. Then for each $u \in \mathbb{R}^{m}$ we have

$$
\max _{v \in \mathcal{D}_{0},\|v\|=1}\langle u, v\rangle=\left\langle u, \frac{P_{D} u}{\left\|P_{D} u\right\|}\right\rangle=\left\|P_{D} u\right\|,
$$

and therefore, by (6.1) and (6.2),

$$
\cos \phi(\lambda)=\max _{u \in \mathcal{A}(\lambda),\|u\|=1}\left\|P_{D} u\right\| .
$$

Since $\|u\|^{2}=\left\|P_{D} u\right\|^{2}+\left\|\left(I-P_{D}\right) u\right\|^{2}$, the last equation can be reformulated as

$$
\cos ^{2} \phi(\lambda)=1-\min _{u \in \mathcal{A}(\lambda),\|u\|=1}\left\|\left(I-P_{D}\right) u\right\|^{2}=1-\sigma(\lambda)^{2}
$$

since $\left(I-P_{D}\right) u$ is just the part of $u$ belonging to the boundary points. By the Pythagorean identity we now have (6.3).

Figure 6.1 shows the situation schematically. In the case of a small subspace angle $\phi(\lambda)$, there exist functions in $\mathcal{A}(\lambda)$ of unit norm that are close to zero on the boundary. These functions are necessarily good approximations of eigenfunctions, as we shall show in Theorem 8.2.

7. Examples. Let us give some examples of the new method in the case of more complex domains, and not just polygons.

Among the most famous polygonal domains are the GWW isospectral drums (Figure 7.1). They are an answer to the question posed by Kac in 1966, "Can One 

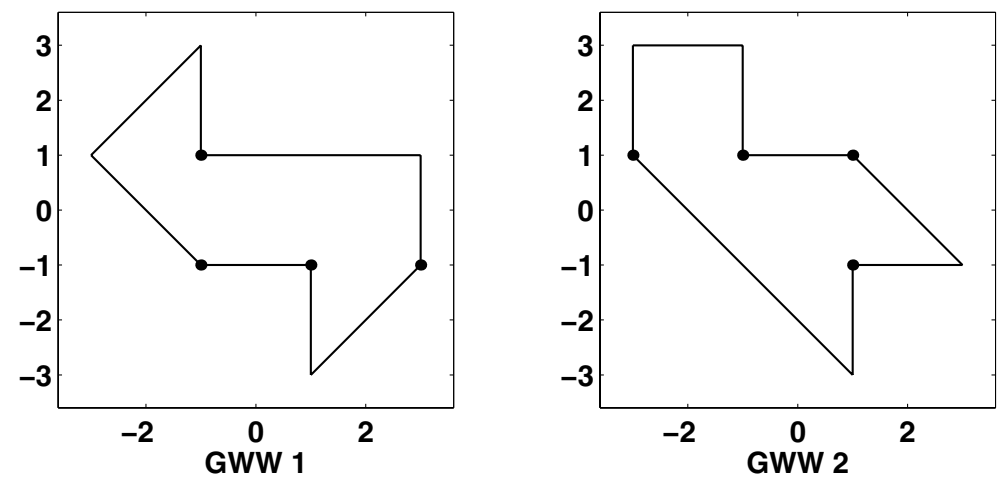

Fig. 7.I The isospectral drums $G W W 1$ and $G W W 2$. The singular corners are marked by dots.

Hear the Shape of a Drum?" [15], which asks whether there exist distinct planar domains on which the Laplacian has the same spectrum. As mentioned earlier, this question remained unanswered until in 1992, Gordon, Webb, and Wolpert constructed pairs of isospectral domains using transplantation techniques [12].

What we call the "GWW drums" are one such pair. The most accurate calculation of eigenvalues of these drums was done by Driscoll [9] with a modified method of Descloux and Tolley [7] that involves domain decomposition and the computation of integrals of Fourier-Bessel functions on the subdomains. Driscoll was able to compute the first 25 eigenvalues to 12 digits. Our modified method of particular solutions achieves the same or better accuracy with a simpler approach. Figure 7.2 shows the subspace angle curves. For all plots, expansions at all the singular corners are used. Although the shapes of the two polygons are distinct, the curves for $\sigma(\lambda)$ are almost identical. With 140 expansion terms at each of the singular corners, 140 boundary points on each side of the polygon and 50 interior points, we obtain the estimates $2.537943999798,3.65550971352$, and 5.17555935622 for the three smallest eigenvalues. For the second and third eigenvalues, all digits agree with the values obtained by Driscoll in [9]. For the first eigenvalue our result appears to be slightly more accurate than Driscoll's estimate of 2.53794399980. The approximate eigenfunctions for the first eigenvalue are shown in Figure 7.3.

Three examples are shown in Figure 7.4. The H-shaped domain was considered by Donnelly in [8] with the original FHM method, using symmetries of the domain. Our modified method, without exploiting symmetries, obtains an approximation for the first eigenvalue of 7.7330888559, which improves Donnelly's result by 5 digits. For the second and third eigenvalues we get 8.5517268486 and 13.9276332229 compared with his 8.55172 and 13.9276 .

In the case of the regular decagon, for the first three eigenvalues, we obtain values of 6.21200099234, 15.76823502672, and 28.31967901332.

For the shape bounded by three quarters of an ellipse, a Fourier-Bessel expansion around the reentrant corner is sufficient to obtain 13 digits. Our approximations for the first three eigenvalues are 5.868746216295, 11.52599695049, and 15.14021979035.

The whole code for plotting $\sigma(\lambda)$, computing the eigenvalues, and plotting the first eigenfunction for a polygon in each case fits on one page and runs in a few seconds if one is willing to settle for, say, 8 digits. Obtaining a random set of interior points is easily done in MATLAB by using the rand function to obtain random points in a 


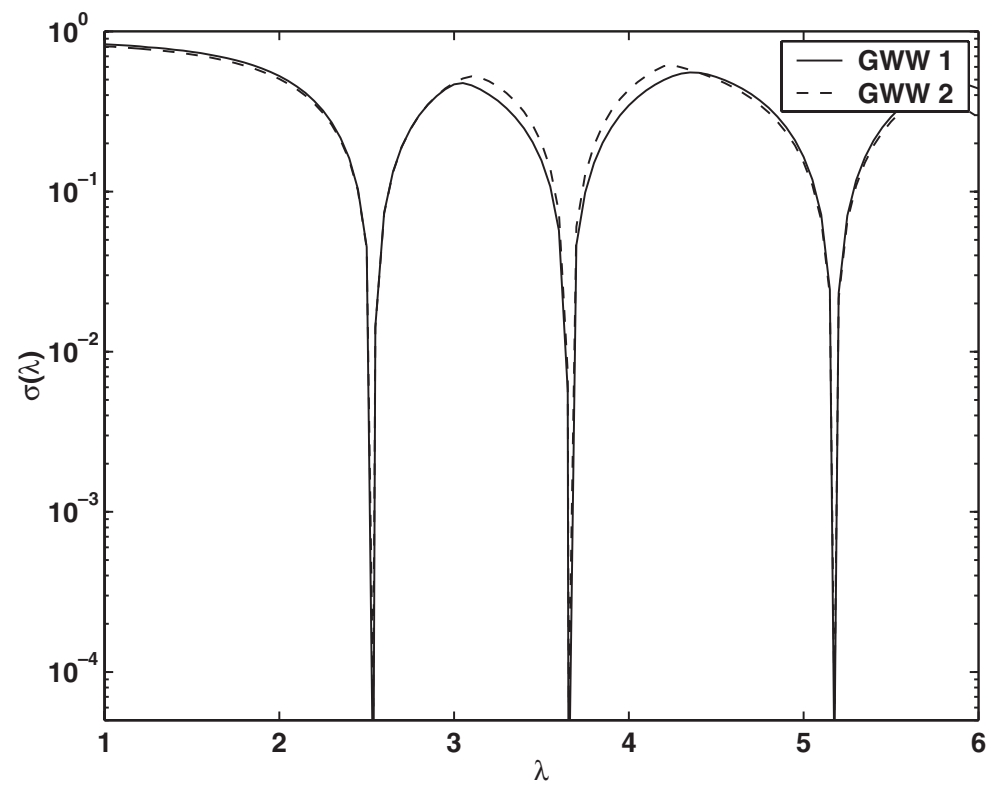

Fig. 7.2 The function $\sigma(\lambda)$ for the isospectral drums $(N=15)$.
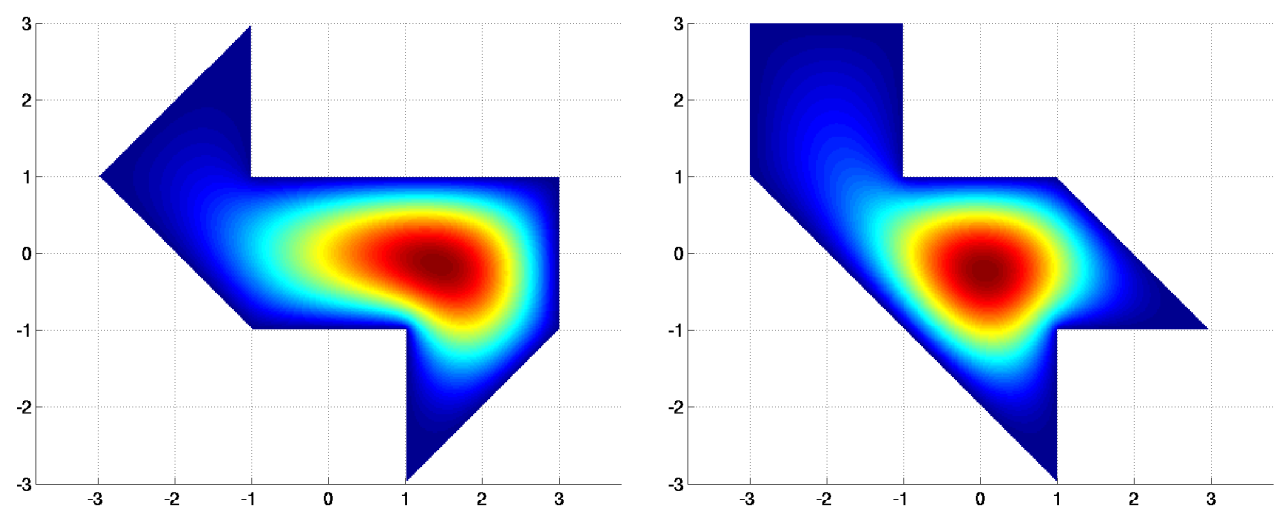

Fig. 7.3 The first eigenfunction on the isospectral drums.

box containing the polygon and then extracting those points in the interior with the function inpolygon.

In the examples presented in this section we used the same number of expansion terms at each corner. In [7] it is proposed to choose the number of expansion terms at each corner in proportion to the interior angle. Hence, a corner with angle $3 \pi / 2$ gets twice as many expansion terms as a corner with angle $3 \pi / 4$. This strategy is sometimes helpful for our new method too.

A numerical study of the phenomenon of "eigenvalue avoidance" carried out by our modified method of particular solutions is presented in [3]. 

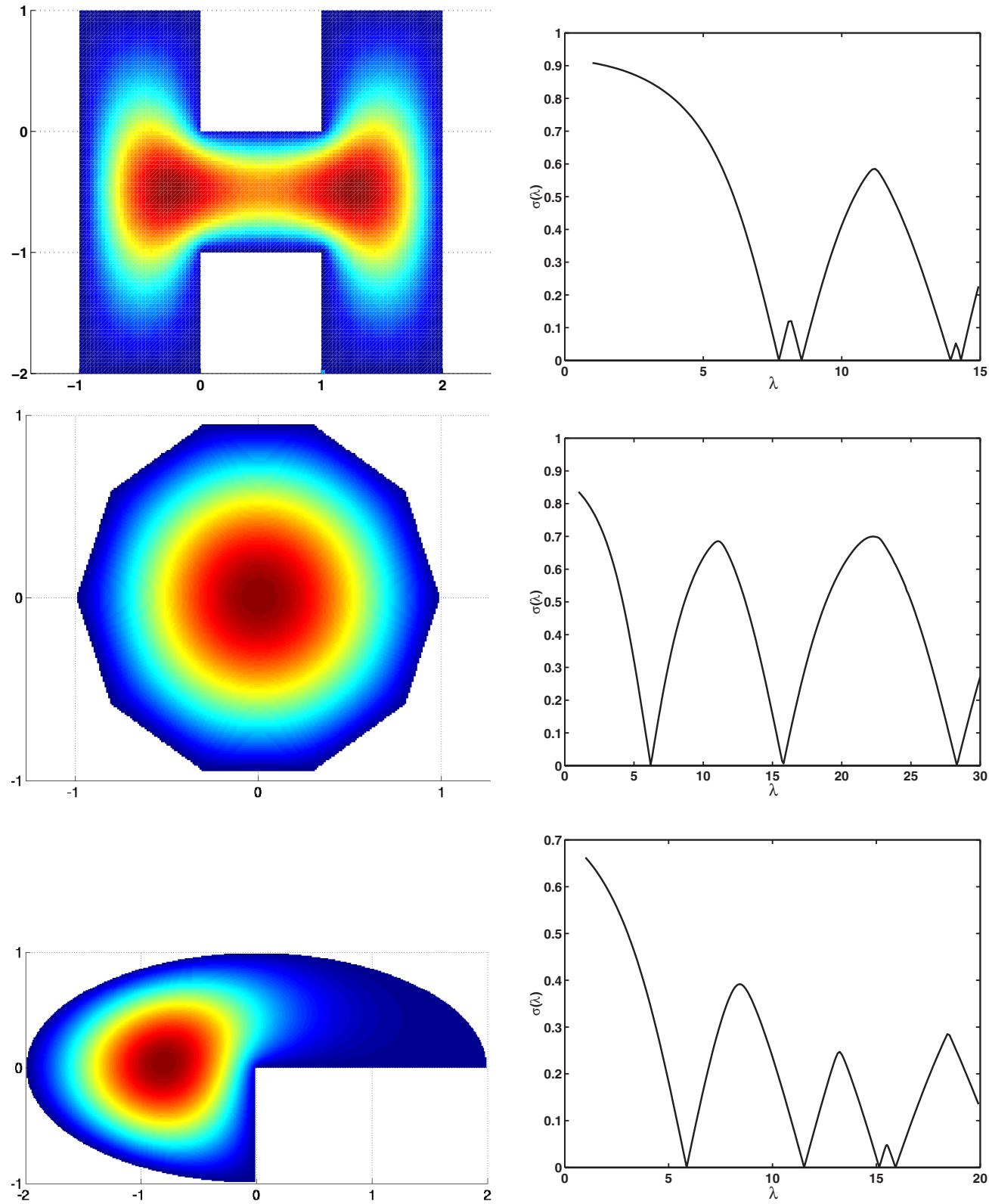

Fig. 7.4 The modified MPS applied to three further domains. The lowest eigenvalues are $\lambda_{1} \approx$ 7.7330888559 (H shape), $\lambda_{1} \approx 6.21200099234$ (decagon), $\lambda_{1} \approx 5.868746216295$ (ellipse minus quadrant). We believe that all the given digits are correct.

8. Error bounds. In this section we review error bounds for the MPS and discuss the application of these bounds to our new method. An excellent overview of error bounds for elliptic eigenvalue problems, including the case of approximate eigenfunctions that do not satisfy the boundary conditions, is given by Still in [27].

In the previous sections the spaces $\mathcal{A}(\lambda)$ and $\mathcal{D}_{0}$ were defined in terms of sample points on the boundary and in the interior of $P$. This definition is no longer sufficient 
for the error bounds presented in this section. Instead, we need the function spaces themselves. Hence, in this section $\mathcal{A}(\lambda)$ is defined as the subspace of $\mathcal{C}^{2}(P) \cap \mathcal{C}(\bar{P})$ of functions that satisfy (2.1a) and $\mathcal{D}_{0}$ is the subspace of $\mathcal{C}^{2}(P) \cap \mathcal{C}(\bar{P})$ of functions that are zero on the boundary. Our norm becomes the area integral

$$
\|u\|:=\left(\int_{P} u^{2} \mathrm{dx}\right)^{1 / 2} .
$$

Our first error bound is an a posteriori result given by Fox, Henrici, and Moler and simplified and extended by Moler and Payne [23].

THEOREM 8.1. Let $\lambda$ and $u$ be an approximate eigenvalue and eigenfunction of (2.1), which satisfy (2.1a) but not necessarily (2.1b). Let $w$ be the harmonic function on $P$ with the same boundary values as $u$, i.e., $w-u \in \mathcal{D}_{0}$, and define

$$
\epsilon:=\|w\| /\|u\| \leq \frac{\sqrt{A} \sup _{x \in \partial P}|u(x)|}{\|u\|},
$$

where $A$ is the area of $P$. Then there exists an eigenvalue $\lambda_{k}$ such that

$$
\frac{\left|\lambda-\lambda_{k}\right|}{\lambda_{k}} \leq \epsilon
$$

If in addition $\|u\|=1$ and $u_{k}$ is the normalized orthogonal projection of $u$ onto the eigenspace of $\lambda_{k}$, then

$$
\left\|u-u_{k}\right\| \leq \frac{\epsilon}{\alpha}\left(1+\frac{\epsilon^{2}}{\alpha^{2}}\right)^{1 / 2},
$$

where

$$
\alpha:=\min _{\lambda_{n} \neq \lambda_{k}} \frac{\left|\lambda_{n}-\lambda\right|}{\lambda_{n}} .
$$

By using this theorem, given any approximate eigenfunction obtained from the MPS, we can derive corresponding error bounds. There is a complication, though, which is that our method uses an orthogonal basis of $\mathcal{A}(\lambda)$ and therefore does not explicitly compute the coefficients $c_{k}^{(N)}$ of (3.2); instead it computes the singular vector $\tilde{v}$ of (5.3). To get the $c$ vector we need to solve the system

$$
A(\lambda) c=Q(\lambda) \tilde{v}
$$

or equivalently

$$
R(\lambda) c=\tilde{v}
$$

where $A(\lambda)=Q(\lambda) R(\lambda)$ is the $Q R$ factorization of $A(\lambda)$. This system is usually highly ill-conditioned, but fortunately, by the general theory of numerical linear algebra, we can expect that although $c$ will be inaccurate, the residual $\|R(\lambda) c-\tilde{v}\|=\| A(\lambda) c-$ $Q(\lambda) \tilde{v} \|$ will nevertheless be small. Thus our computed $A(\lambda) c$ will be very small at the sample points on $\partial P$, and experiments show it is small on the rest of $\partial P$ too. It is this magnitude over all of $\partial P$ that we need to measure to apply Theorem 8.1.

Let us demonstrate this for the L-shaped domain. Fox, Henrici, and Moler gave bounds for the first 10 eigenvalues and showed that their approximation for the first 


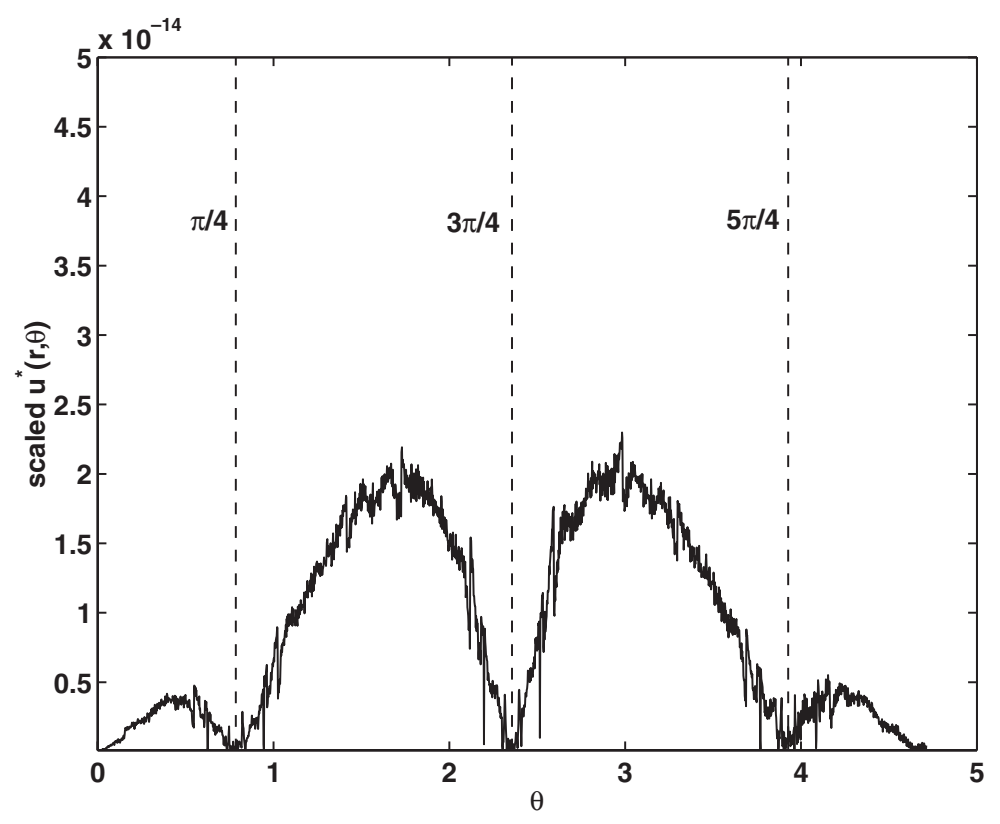

Fig. 8.I Numerically computed magnitude of the approximate eigenfunction $u^{*}(r, \theta)$ along the boundary of the $L$ shape after scaling by the square root of the area and the norm of $u^{*}$. Dashed lines mark the corners of the domain. Rounding errors lead to oscillations around the true function values.

eigenvalue was correct to at least 8 digits [11]. We find that at least the first 13 rounded digits of our approximation 9.6397238440219 are correct. In this computation we used 100 Chebyshev-distributed points on each side of the boundary nonadjacent to the reentrant corner and 500 interior points. The use of Chebyshev points has the effect that the approximate eigenfunction (3.2) stays small near the corners. With equally spaced points a high peak of (3.2) appears at the corner opposite the reentrant corner, resulting in poor error bounds.

Even with a condition number of $R(\lambda)$ on the order of $10^{76}$, the values of $A(\lambda) c$ differ from the values $Q(\lambda) \tilde{v}$ at the interior points only by componentwise relative errors on the order of $10^{-13}$. At the boundary points the values differ significantly, but the norms of $A_{B}(\lambda) c$ and $Q_{B}(\lambda) \tilde{v}$ are $5.0459 \times 10^{-15}$ and $5.0464 \times 10^{-15}$. Thus the function $u^{*}$ we have constructed in the form (3.2) is a good approximate eigenfunction.

A lower bound for the $L_{2}$ norm of $u^{*}$ can be computed by integrating only over the lower left unit square of the L-shaped domain. This ensures that errors introduced by the MATLAB function dblquad don't lead to an approximation of $\left\|u^{*}\right\|$ that is larger than the true value. Figure 8.1 shows the values of $u^{*}$ along the boundary scaled by this lower bound of $\left\|u^{*}\right\|$ and the square root of the area of the domain. The oscillations of the curve are caused by rounding errors. Since these oscillations also lead to values that are larger than the true values of $u^{*}$, we can still obtain a good upper bound for $u^{*}$. Finding the maximum of this curve, we obtain

$$
9.6397238440216 \leq \lambda_{1} \leq 9.6397238440222
$$

compared to our approximation 9.6397238440219. Therefore, at least 13 rounded 
digits are correct. The curve in Figure 5.3 suggests that actually all 14 digits are possibly correct, and indeed, we think that the 15th digit is either 4 or 5 .

The bounds we have been discussing start from an approximate eigenfunction obtained from the MPS. However, what if we go back a step and work with the subspace angle $\phi(\lambda)$ itself? In previous sections, as in the algorithm as implemented in practice, $\phi(\lambda)$ was defined in terms of the sampled spaces $\mathcal{A}(\lambda)$ and $\mathcal{D}_{0}$, and from here, no bound comes readily to hand. However, let us imagine instead that $\phi(\lambda)$ is defined by (6.2) in terms of the continuous rather than sampled spaces $\mathcal{A}(\lambda)$ and $\mathcal{D}_{0}$ introduced at the beginning of this section. Now we obtain a clean theorem showing that if $\phi(\lambda)$ is small, $\lambda$ is close to an eigenvalue.

TheOREM 8.2. Let $\mathcal{A}(\lambda)$ and $\mathcal{D}_{0}$ be defined as in the beginning of this section and define the inner product $\langle u, v\rangle:=\int_{P} u v d x+\int_{\partial P} u v d x$ with corresponding norm $|\|u\||:=\sqrt{\langle u, u\rangle}$. Then with the subspace angle $\phi(\lambda)$ between $\mathcal{A}(\lambda)$ and $\mathcal{D}_{0}$ defined by (6.1) and (6.2), there exists an eigenvalue $\lambda_{k}$ such that

$$
\frac{\left|\lambda-\lambda_{k}\right|}{\lambda_{k}} \leq c \tan \phi(\lambda)
$$

where $c>0$ depends only on the domain $P$. Furthermore, for every $\delta>0$ and $\tilde{\epsilon}:=c \tan \phi$ there exists a function $u \in \mathcal{A}(\lambda),\|u\|=1$ such that

$$
\left\|u-u_{k}\right\| \leq \frac{\tilde{\epsilon}}{\alpha}\left(1+\frac{\tilde{\epsilon}^{2}}{\alpha^{2}}\right)^{1 / 2}+\delta,
$$

where $\alpha$ and $u_{k}$ are defined as in Theorem 8.1.

Proof. For every $u \in \mathcal{A}(\lambda)$ we have

$$
\sup _{\substack{v \in \mathcal{D}_{0} \\ \mid\|v\| l=1}}\langle u, v\rangle=\|u\|
$$

since from $\langle u, v\rangle=\int_{P} u v \mathrm{dx}$ for every $v \in \mathcal{D}_{0}$ and the Cauchy-Schwarz inequality it follows that $\langle u, v\rangle \leq\|u\|$ for every $v \in \mathcal{D}_{0}$ with $\mid\|v\| \|=1$. Equality in (8.7) follows from the fact that $u$ can be expanded in $P$ in terms of the eigenfunctions $v_{k} \in \mathcal{D}_{0}$ of (2.1). Combining (6.1), (6.2), and (8.7), we get

$$
\cos \phi(\lambda)=\sup _{\substack{u \in \mathcal{A}(\lambda) \\\|\| u \|=1}}\|u\|
$$

For every $u \in \mathcal{A}(\lambda)$ there exists a function $w$ that is harmonic in $P$ with $u=w$ on $\partial P$. Since $w$ is harmonic, there is a constant $c>0$ that depends only on $P$ such that $\|w\| \leq c \sqrt{\int_{\partial P} w^{2} \mathrm{dx}} \cdot{ }^{3}$ If $|\|u\||=1$, then

$$
\epsilon:=\frac{\|w\|}{\|u\|} \leq c \frac{\sqrt{1-\|u\|^{2}}}{\|u\|} .
$$

From (8.8) it follows that

$$
\inf _{\substack{u \in \mathcal{A}(\lambda) \\\|u\| l=1}} \frac{\sqrt{1-\|u\|^{2}}}{\|u\|}=\tan \phi(\lambda),
$$

\footnotetext{
${ }^{3} \mathrm{~A}$ discussion of this constant can be found in [16].
} 
which together with (8.1) and (8.9) results in (8.5). Equation (8.6) follows from (8.2) by choosing $u$ sufficiently close to this infimum.

Theorem 8.2 is different from Theorem 8.1 in that it does not only give bounds based on a given approximate eigenfunction. Instead it answers the question of how well an eigenvalue and eigenvector can be approximated from the whole space $\mathcal{A}(\lambda)$. This is a stronger result. The angle $\phi(\lambda)$ between the continuous spaces $\mathcal{A}(\lambda)$ and $\mathcal{D}_{0}$ cannot be directly computed, but by choosing many interior and boundary sample points we get good approximations to it.

9. Discussion. We have shown that the MPS can be an accurate and reliable means of approximating eigenvalues and eigenfunctions on polygonal and other simple domains. The examples in section 7 demonstrate the effectiveness of the new method even in the case of several singularities. Figure 9.1 presents a short MATLAB code that computes the first three eigenvalues of the $\mathrm{L}$ shape to 10 digits of accuracy in less than five seconds on a Pentium 4 system.

In our experiments the method behaves robustly with respect to different choices of interior points and boundary points. Usually we take the boundary points to be equally distributed. Sometimes a Chebyshev distribution is preferable, a change of one line in a MATLAB code. A random distribution of interior points has always proved effective. In principle the method would fail if all points fell in regions where the eigenfunction is close to zero, but this is easily prevented by taking a healthy number of randomly distributed points. For the speed of the algorithm there is not much difference between 50 or 500 interior points; the work in both linear algebra and evaluation of Bessel functions scales just linearly with respect to $m_{I}$. Theorem 8.1 shows that the original bounds expanded by Fox, Henrici, and Moler and improved by Moler and Payne are also applicable to the new method, which, with a careful implementation, allows reliable a posteriori bounds on almost arbitrary polygons. Theorem 8.2 shows that there is a direct link from subspace angles to accuracy.

The only limitation of the robustness of the method that we have encountered occurs mainly when, in addition to expansions at singular corners, expansions at regular corners are added. The Fourier-Bessel basis then contains redundant information that leads to arbitrary columns of $Q$ in the $\mathrm{QR}$ factorization, causing oscillations in $\sigma(\lambda)$ and making it difficult to locate the eigenvalues by automatic minimization. This problem can be largely solved by using a column-pivoted QR factorization and discarding columns of $Q$ that correspond to very small elements in $R$. In Figures 7.2 and 7.4b we have done this, as well as in Figure 9.3 below. However, with this strategy there seems to be a loss in attainable accuracy. In cases with expansions at only the singular corners, the oscillations were small in most of our experiments and never disturbed the detection of eigenvalues.

Most of the time, we use minimizers like the MATLAB function fminsearch to find the minima of $\sigma(\lambda)$. The code in Figure 9.1 uses a different approach. It follows the question, "What if we had signed subspace angles?" If $\sigma(\lambda)$ attains a local minimum of exactly zero, we can redefine $\sigma(\lambda)$ to have a sign change there, whereupon looking for eigenvalues becomes a problem of zerofinding rather than minimization. The same idea works in practice when $\sigma(\lambda)$ has a local minimum that is very small but nonzero. The reader may enjoy downloading this program and giving it a try. However, we do not have a theory of signed subspace angles that encompasses these "almost-but-not-quite" situations. It would be interesting to pursue this idea.

Another generalization of the $\sigma(\lambda)$ function would be to consider its behavior in the complex $\lambda$-plane. For example, Figure 9.2 shows level curves $|\sigma(\lambda)|=$ 
$\%$ Ldrum.m - eigenvalues of Laplacian on L-shaped region

$\%$

$\%$ The first 3 eigenvalues are computed by the method of

$\%$ particular solutions, using interior as well as boundary

$\%$ points. T. Betcke and L.N. Trefethen, 2003.

$\%$ Compute subspace angles for various values of lambda:

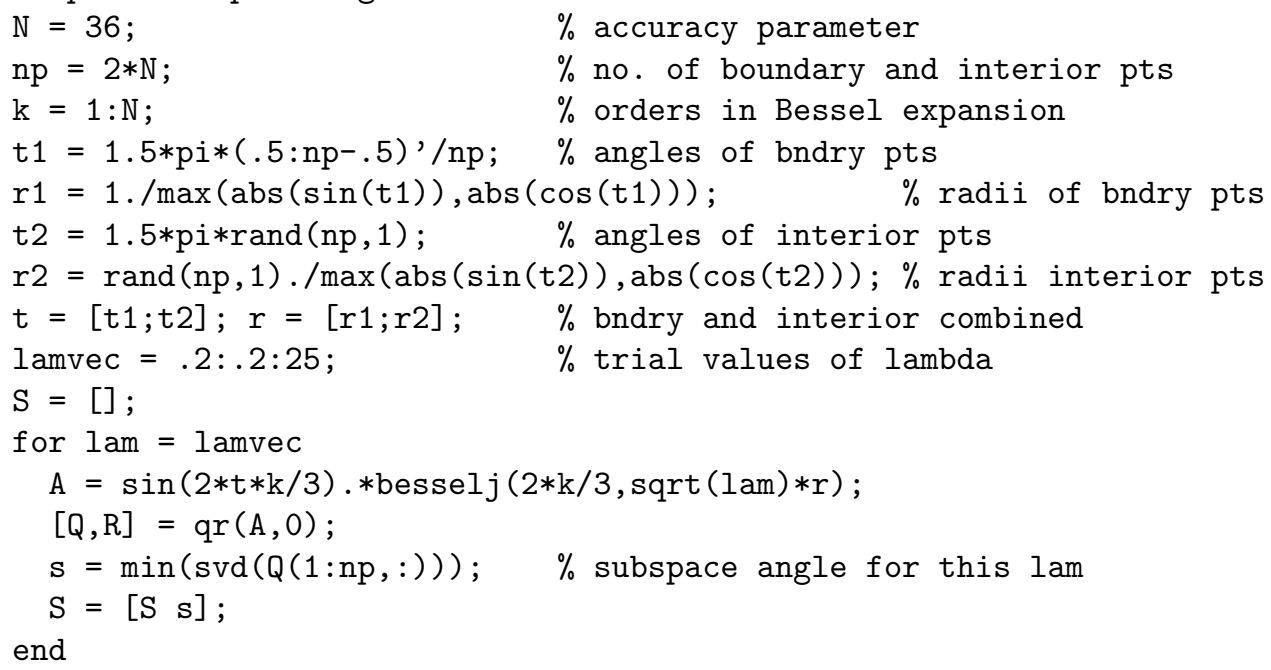

Fig. 9.I This short MATLAB code computes the first three eigenvalues of the L-shaped domain to 10 digits of accuracy in less than five seconds on a Pentium 4 with a speed of 1.8 Ghz. It is available online at http://www.comlab.ox.ac.uk/work/nick.trefethen.

$0.05,0.1,0.15, \ldots$ in a rectangular subset of the plane with $N=15$. Figure 5.2 can be interpreted as a cross-section through this figure. It may seem odd to go into the complex plane for a self-adjoint problem like this, but the same point of view has been used in [30] to obtain bounds for the convergence of Lanczos iterations for eigenvalues of hermitian matrices. The context of [30] is pseudospectra of rectangular 


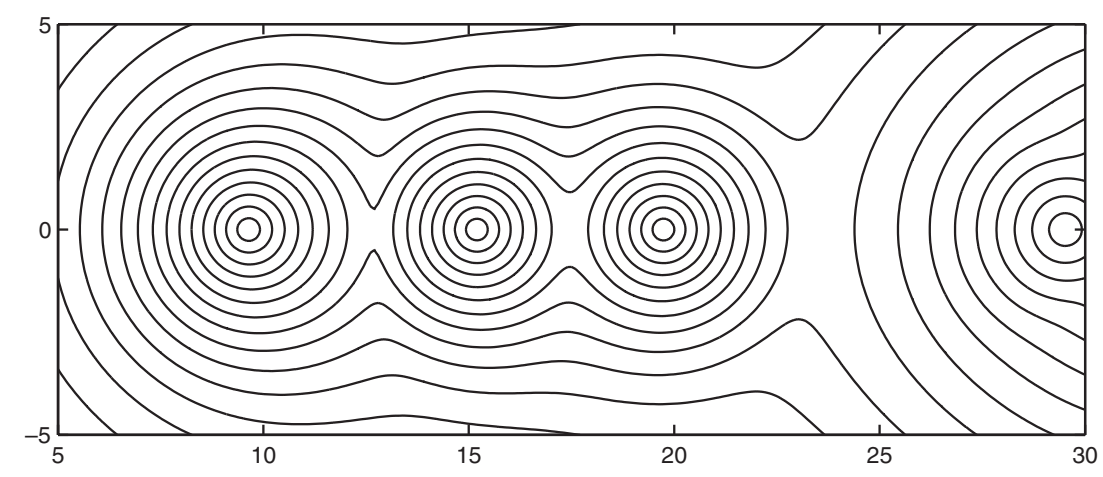

Fig. 9.2 Extension of Figure 5.2 into the complex $\lambda$-plane: level curves $|\sigma(\lambda)|=0.05,0.1,0.15, \ldots$ for the L-shaped region with $N=15$.

matrices, and indeed, Figure 9.2 could be interpreted as a plot of pseudospectra for a rectangular nonlinear eigenvalue problem. Our Theorem 8.2 is related to the bounds given in [30] for Lanczos and Arnoldi iterations, which are in turn related to further decades-old work by Lehmann.

Another related method of approximating eigenvalues can be found in a recent paper on "spectral pollution" by Davies and Plum [6]. These authors also view eigenvalues as minimal points of certain curves and obtain bounds by looking at the curves corresponding to finite-dimensional subspaces. Figure 1 of [6] will look familiar to any reader of this paper.

Our method minimizes $\sigma(\lambda)$, the sine of the angle between $\mathcal{A}(\lambda)$ and $\mathcal{D}_{0}$. But the other principal angles between these two subspaces also show interesting behavior. The $k$ th principal angle $\theta_{k}$ between spaces $\mathcal{F}$ and $\mathcal{G}$ is defined recursively by

$$
\cos \theta_{k}:=\left\langle u_{k}, v_{k}\right\rangle=\sup _{\substack{u \in \mathcal{F} \\ v \in \mathcal{G},\|u\|=1 \\\|v\|=1}}\langle u, v\rangle, \quad u \perp u_{1}, \ldots, u_{k-1}, \quad v \perp v_{1}, \ldots, v_{k-1},
$$

where the pairs $\left(u_{j}, v_{j}\right), j=1, \ldots, k-1$, are the principal vectors associated with $\theta_{1}, \ldots, \theta_{k-1}$. The sines of the principal angles are the singular values of $Q_{B}(\lambda)$; details can be found in [4]. Figure 9.3 shows these first three singular values as functions of $\lambda$ in the case of the isospectral drum GWW 1. It is apparent that the maxima of the first principal angle correspond to minima of the second principal angle, with analogous behavior shown by the second and third principal angles. It would be interesting to investigate the implications of this behavior.

In this paper we have not addressed the question of the approximation quality of the Fourier-Bessel spaces. With expansions at least at all singular corners we observe spectral convergence in our experiments, as in Figure 5.3. In the case of one singular corner this behavior can be verified by canceling out the singularity with a conformal mapping and using convergence estimates from complex analysis to obtain spectral convergence. If the domain has several singular corners, however, the situation is more complicated. These issues will be dealt with in a separate paper.

In closing we want to emphasize that our method is applicable to a wider class of problems than the eigenvalues of the Laplacian with Dirichlet boundary conditions. The idea of minimizing the angle between the subspaces satisfying the PDE and the boundary conditions is a general one, applicable to all kinds of problems in which particular solutions are available. Extensions of our method to eigenvalue calculations 


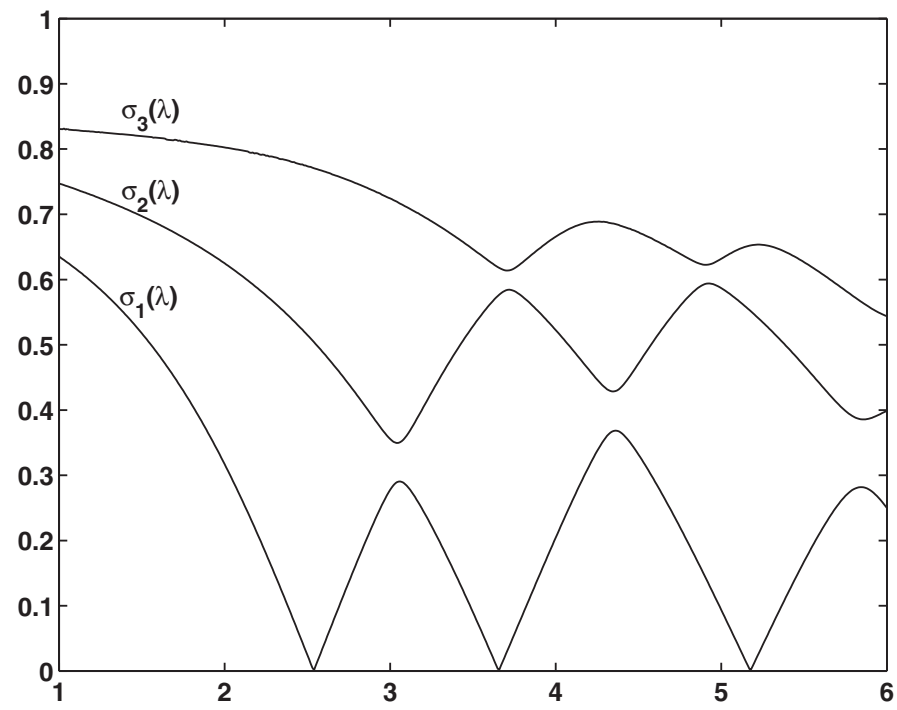

Fig. 9.3 The sines of the first three principal angles for the isospectral drum $G W W 1$.

in three space dimensions and to other problems are under investigation. Related ideas are also being found to be useful in the finite elements literature, e.g., in the partition of unity finite element method (PUFEM) method of Melenk and Babuška [20].

Note Added in Proof. Two important developments have occurred since this article was first accepted for publication. First, connections have been developed, to be reported elsewhere, with the generalized singular value decomposition, the general framework for the most stable algorithms for computing subspace angles. We have also learned that in unpublished work going back to the 1970s, Stanley Eisenstat anticipated a number of the ideas we have presented and also considered the use of the GSVD. Second, we have become aware of the large and remarkable body of work on numerical solution of planar eigenvalue problems by physicists concerned with quantum chaos and related matters, including Heller [13], Vergini and Saraceno [29], and Barnett [1]. Where our emphasis is on high accuracy and treatment of corner singularities, theirs is on behavior of high eigenmodes. Their algorithmic innovations are very significant, and efforts are underway to synthesize the ideas of these heretofore largely disjoint literatures.

Acknowledgments. The comments and suggestions of Toby Driscoll and Cleve Moler have been invaluable for us in the course of this work. We are also grateful for advice from Lance Bode, Dominic Donnelly, Gene Golub, Wayne Read, Graeme Sneddon, and Gilbert Strang. Read, Sneddon, and Bode have also recently been making use of the method of particular solutions [24].

\section{REFERENCES}

[1] A. H. Barnett, Dissipation in Deforming Chaotic Billiards, Ph.D. thesis, Department of Physics, Harvard University, Cambridge, MA, 2000.

[2] S. Bergman, Functions satisfying certain partial differential equations of elliptic type and their representation, Duke Math. J., 14 (1947), pp. 349-366.

[3] T. Betcke And L. N. Trefethen, Computations of eigenvalue avoidance in planar domains, Proc. Appl. Math. Mech., 4 (2004), pp. 634-635. 
[4] A. Buörck AND G. H. Golub, Numerical methods for computing angles between subspaces, Math. Comp., 27 (1973), pp. 579-594.

[5] R. Courant And D. Hilbert, Methods of Mathematical Physics, Vol. I, Interscience, New York, 1953.

[6] E. B. Davies And M. Plum, Spectral pollution, IMA J. Numer. Anal., 24 (2004), pp. 417-438.

[7] J. Descloux AND M. Tolley, An accurate algorithm for computing the eigenvalues of a polygonal membrane, Comput. Methods Appl. Mech. Engrg., 39 (1983), pp. 37-53.

[8] J. D. P. Donnelly, Eigenvalues of membranes with reentrant corners, SIAM J. Numer. Anal., 6 (1969), pp. 47-61.

[9] T. A. Driscoll, Eigenmodes of isospectral drums, SIAM Rev., 39 (1997), pp. 1-17.

[10] S. C. Eisenstat, On the rate of convergence of the Bergman-Vekua method for the numerical solution of elliptic boundary value problems, SIAM J. Numer. Anal., 11 (1974), pp. 654680.

[11] L. Fox, P. Henrici, And C. B. Moler, Approximations and bounds for eigenvalues of elliptic operators, SIAM J. Numer. Anal., 4 (1967), pp. 89-102.

[12] C. Gordon, G. WebB, And S. Wolpert, Isospectral plane domains and surfaces via Riemannian orbifolds, Invent. Math., 110 (1992), pp. 1-22.

[13] E. J. Heller, Wavepacket dynamics and quantum chaology, in Proceedings of the 1989 Les Houches Summer School on Chaos and Quantum Physics, M. J. Giannoni, A. Voros, and J. Zinn-Justin, eds., Elsevier, 1991, pp. 547-663.

[14] J. Hersch, Erweiterte Symmetrieeigenschaften von Lösungen gewisser linearer Rand- und Eigenwertprobleme, J. Reine Angew. Math., 218 (1965), pp. 143-158.

[15] M. KAC, Can one hear the shape of a drum?, Amer. Math. Monthly, 73 part II (1966), pp. $1-23$.

[16] J. R. Kuttler, Remarks on a Stekloff eigenvalue problem, SIAM J. Numer. Anal., 9 (1972), pp. 1-5.

[17] J. R. Kuttler and V. G. Sigillito, Eigenvalues of the Laplacian in two dimensions, SIAM Rev., 26 (1984), pp. 163-193.

[18] R. S. Lehmann, Developments at an analytic corner of solutions of elliptic partial differential equations, J. Math. Mech., 8 (1959), pp. 729-760.

[19] J. C. MASOn, Chebyshev polynomial approximations for the L-membrane eigenvalue problem, SIAM J. Appl. Math., 15 (1967), pp. 172-186.

[20] J. M. MeLenK AND I. BABUŠKA, Approximation with harmonic and generalized harmonic polynomials in the partition of unity method, Comput. Assist. Mech. Eng. Sci., 4 (1997), pp. $607-632$.

[21] C. B. Moler, Accurate Bounds for the Eigenvalues of the Laplacian and Applications to Rhombical Domains, Report CS-TR-69-121, Department of Computer Science, Stanford University, Stanford, CA, 1969.

[22] C. B. Moler, Numerical Computing with MATLAB, SIAM, Philadelphia, 2004.

[23] C. B. Moler And L. E. PaYne, Bounds for eigenvalues and eigenfunctions of symmetric operators, SIAM J. Numer. Anal., 5 (1968), pp. 64-70.

[24] W. W. Read, G. E. Sneddon, and L. Bode, A seried method for the eigenvalues of the advection diffusion equation, ANZIAM J., 45 (2004), pp. C773-C786.

[25] J. K. ReID AND J. E. WALSh, An elliptic eigenvalue problem for a reentrant region, SIAM J. Appl. Math., 13 (1965), pp. 837-850.

[26] N. L. Schryer, Constructive approximation of solutions to linear elliptic boundary value problems, SIAM J. Numer. Anal., 9 (1972), pp. 546-572.

[27] G. STILl, Computable bounds for eigenvalues and eigenfunctions of elliptic differential operators, Numer. Math., 54 (1988), pp. 201-223.

[28] I. N. VeKUA, Novye metody rešenija elliptičkikh uravnenij (New Methods for Solving Elliptic Equations), OGIZ, Moskow and Leningrad 1948.

[29] E. Vergini and M. Saraceno, Calculation by scaling of highly excited states of billiards, Phys. Rev. E, 52 (1995), pp. 2204-2207.

[30] T. G. Wright and L. N. Trefethen, Pseudospectra of rectangular matrices, IMA J. Numer. Anal., 22 (2002), pp. 501-519. 